\title{
Kinetics and mechanism of $\alpha$-glucosidase inhibition by edible brown algae in the management of type 2 diabetes
}

\section{Abstract}

Prevalence of type 2 diabetes mellitus has significantly increased in the last three decades and currently affects about 1 in 10 globally. A common therapeutic target for type 2 diabetes is $\alpha$ - glucosidase, an essential enzyme located at the brush border of the small intestinal epithelium. The inhibition of $\alpha$-glucosidase results in a reduced digestion of carbohydrates and a decrease of postprandial blood glucose. Although, synthetic inhibitors are available in the market, these are usually associated with significant gastrointestinal side effects. In this study, natural inhibitors derived from edible brown algae are being investigated as an alternative.

Polysaccharide- and polyphenolic-enriched extracts from Ascophyllum nodosum and Fucus vesiculosus were characterized and screened for their inhibitory effects against $\alpha$-glucosidase obtained from rat intestine using maltose, sucrose, and p-nitrophenyl (pNPG) as substrates. Acarbose was used as a synthetic inhibitor. Furthermore, enzyme kinetics and mechanism of inhibition of $\alpha$ - glucosidase were determined using linear and non-linear regression methods (GraphPad Prism ver. 6, GraphPad Software, La Jolla California USA).

All tested extracts showed a dose-dependent inhibitory effect against $\alpha$-glucosidase. However, the type of inhibition varied between the extracts. Most importantly, the composition analysis showed that the seaweed extracts had different polysaccharide and phenolic contents, suggesting different mode of actions against $\alpha$-glucosidase. The relation between chemical composition and inhibitory activity of the compounds are discussed.

In summary, the current study demonstrates the mechanisms in which different brown seaweed extracts with various composition effectively inhibit $\alpha$-glucosidase. Therefore, this natural inhibitor can be considered as a potential candidate for the management of type 2 diabetes mellitus.

\section{Conflict of Interest}

Shane O'Connell and Sinead Ryan are employees of Marigot Ltd 